MATEC Web of Conferences 40, 07011 (2016)

DOI: $10.1051 /$ matecconf/20164007011

(c) Owned by the authors, published by EDP Sciences, 2016

\title{
A New Smart Grid Control and Operation Concept - Autonomic Power System
}

\author{
Bo Gao ${ }^{1,2,3}$, Guangyi $\mathrm{Liu}^{2}$, Yanbin $\mathrm{Qu}^{3}$, Zhanyong Yang ${ }^{2}$, Shixiong Fan ${ }^{2}$, Yang Yang ${ }^{2}$, Kai Zhang ${ }^{4}$, Bin Shu ${ }^{4}$, Tong \\ Zhou $^{4}$ \\ ${ }^{1}$ Harbin Institute of Technology, Harbin, China; \\ ${ }^{2}$ China Electric Power Research Institute, Beijing, China; \\ ${ }^{3}$ Harbin Institute of Technology at Weihai, Weihai, China; \\ ${ }^{4}$ Beijing Electric Power Economy Research Institute, Beijing, China
}

\begin{abstract}
As the distributed energy resource (DER) penetrance increasing, future power system will be more largescale with much complexity and uncertainty. Taking an active approach, active distribution network (ADN) can deal with future complex and uncertain challenges to some extent. However, too much re-lying on information exchange of each layer, ADN will lack global adaptability and stability. To make up for the deficiency, this paper proposes a new smart grid control and operation concept, namely autonomic power system (APS). Based on goal-directed mechanism, APS obtains global self-adaptive management. Meanwhile, under the concept of distributed coordination and autonomic control, autonomic units (AUs) in APS coordinate each other and make their own autonomic operation. APS takes not only an optimally and co-ordinately active control of power system, but also brings more intelligence with characteristics of self-configuration, self-optimization, self-protection and self-healing, i.e. self-management and overall self-adaption. Thus, APS completes real-time dynamic goals and makes the operation of future power system more intelligent, effective, safe and reliable.
\end{abstract}

\section{Introduction}

As the issue on environment and energy more and more serious, much more distributed energy resource (DER) will be used in power system in the future with more complexity and uncertainty at the same time [1]. It makes traditional passive control and operation technology unsuitable for the future power system. Thus active distribution network (ADN) is proposed and obtains increasing attention [2]. Taking an active approach in distribution network management, ADN can improve the system controllability, coordination, and DER penetrance [3]. However, too much depending on information interaction among levels, ADN lacks overall adaptability not well prepared for future challenges derived from DER connection.

In recent years, autonomic computing (AC) [4] proposed by IBM attracts many scholars' attention. It highlights the concept of distributed autonomic management by resolving large-scale system problems into series self-management issues. Having made success in autonomic computing system (ACS) [5], it gives new idea in solving real-time decision making in complex system. This self-adaptive concept is also similar with system of systems (SoS) [6]. It reflects that complex systems need inner self-management to keep stable, and the power system is no exception. Intelligent control of autonomic computing (AC) for complex systems offers a new way in solving future power system complexity and uncertainty. The primary challenges of future power system are dealing with complexities of coordinating and managing a large set of diverse system components and functions including distributed generations (DGs), storage devices, system faults and power restoration management, energy brokerage and trading, and consumer demand power management. To meet these challenges, it is required to extend the computer science community's vision of autonomic computing into the electricity network for energy innovation.

Actually, from initial distribution automation (DA) to advanced grid operation control technology, such as multi agent system (MAS) [7], active network management (ANM) [8], these all include self-management concept to be more autonomic. Recently some new smart grid concepts are introduced based on autonomic control, such as micro-grid island autonomy [9], autonomous demand area power system (ADAPS) [10], intelligent distributed autonomous power systems (IDAPS) [11], and autonomous regional active network management system (AuRA-NMS) [12]. As early exploration of autonomic power system, these smart grid concepts contain autonomic operation control technologies including selfhealing and self-optimization. Especially based on MAS and ANM, AuRA-NMS has achieved some success in self-healing, but it is not enough for future challenges. With power system be more intelligent, there will be 
more autonomic basis from units to regions. Single smart devices and local networks will be able to solve problems autonomously, and establish interaction communication protocol between higher and lower level as needed. These autonomic mechanisms fully embody hierarchical autonomous smart grid concept, greatly reducing the toplevel control burden and information exchange. Thus future smart grid should be autonomic in low-level as well as controllable in top-level, coordinated in middle level. In this way, it can deal with all possible cases including normal or faulted conditions. This paper proposes a stronger and smarter grid concept, namely autonomic power systems (APS). Based on autonomic control and operation, APS can effectively respond to future challenges and free complex system operation problems out. This paper will introduce APS control and operation mechanism in detail, especially on its control and operation architecture.

The structure of this paper is as followings. After autonomic power system (APS) control and operation concept illustrated in part II, part III designs and analyzes APS control and operation architecture. Part IV concludes the paper and discusses further research perspectives.

\section{Autonomic concept}

In the perspective of control and operation, global selfadaptive management and distributed self-management compose two impartible cores in APS. These two points are mainly embodied by goal-directed mechanism, dynamic control zones division and four autonomic characteristics.

\subsection{Goal-directed Mechanism}

Goal-directed mechanism is a novel concept of great importance for APS overall adaptability. Based on goaldirected mechanism and dynamic control zone concept, autonomic operation and control overall structure is illustrated in figure 1 .

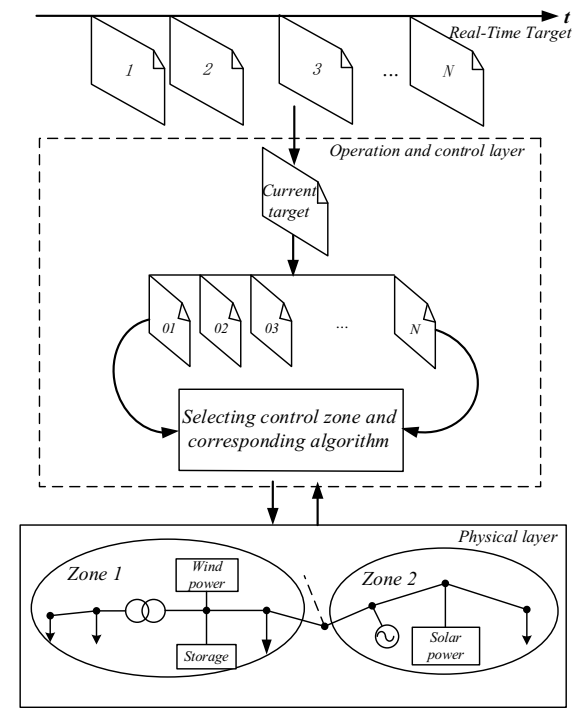

Figure 1. Autonomic operation and control structure
When current top-level goal is generated, a series of sub-goals are generated by intelligent decomposition for corresponding control zones and will be accomplished respectively. Top-level goal is identified by power system control center, such as ensuring a number of electric cars ready for charging. Then it will be divided into specific sub-goals. As for above example, sub-objectives may be maintaining voltage within certain limits and assigning a certain power for electric cars and so on. Then Autonomic management works after appropriate control domains and corresponding algorithms identified. It will be a self-management process of no external interference. Based on this mechanism, APS overall adaptability is fulfilled by distributed cooperation of control domains.

\subsection{Dynamic Control Zones Division}

Depending on sub-goals generated by goal-directed mechanism and physical system operation conditions such as fault occurring or network topology variation, dynamic control zones division and algorithms mapping mechanism is designed as shown in figure 2 .



Figure 2. Dynamic control zones division and algorithms mapping mechanism

In control zones, autonomic region boundaries are adjusted as well as mapped with corresponding control algorithms by intelligent control algorithm allocation center. This will be easily realized by high speed computer in the future. Coordination such as exchanging border power and sharing constraints is carried out between two adjacent zones with their boundaries dynamically be expanded, shrunken, merged or decomposed. Here, the control zones in APS are chosen dynamically with flexible and dynamic boundaries depending on current real-time control objectives. It is totally different from zones in traditional power systems that are statically made independent control areas in hierarchical control structure. This difference makes autonomic power system be more flexible, optimal and efficient to choose appropriate domains to accomplish sub-goals. This process is predictive, preventive and autonomic.

Based on goal-directed mechanism and dynamic control zones division in APS, high-level goals change dynamically and are transferred into local region objectives. Then they are achieved automatically and coordinately in each flexible and optimal sub-domain with a dynamic control range generated as needed. 


\subsection{Autonomic Characteristics}

Self-management of APS is embodied by four characteristics namely self-configuration, selfoptimization, self-protection and self-healing. As shown in figure 3, as a whole these four specialties do reflect APS autonomic concept in all operation conditions. Each of them has different functions, but they interact in some way when needed.

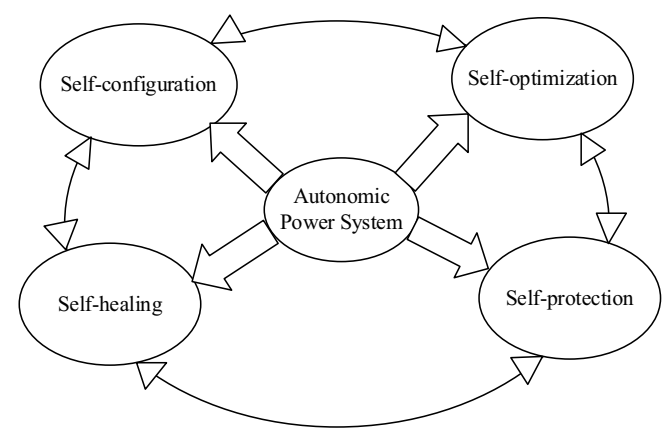

Figure 3. APS characteristics

\subsubsection{Self-configuration}

Self-configuration is the ability to self-adapt to dynamically changing environments based on preestablished operations knowledge. Self-configuration systems can determine current time optimal operating configuration automatically in reaction to system perturbations or as a response to self-optimization, selfprotection and self-healing. It is the basis of other three characteristics. Based on advanced information and communication technology (AICT) for real-time information of power system status and intelligent analysis on environmental changes, power system resources are self-reconfigured to meet changing environment, which makes APS a strong adaptability and robustness.

\subsubsection{Self-optimization}

Self-optimization is the ability to monitor and tune resources automatically to meet system goals. Attributes information such as component performance, security, availability, and reliability are required. Based on system configuration and operational status, using real-time dynamic intelligent optimization algorithm, selfoptimization improves system performance to achieve the best use of available resources, and maintains the efficient operation of the system. It will maximize the use of renewable energy sources, reduce power line losses and combine goals depending on the goals set in the APS.

\subsubsection{Self-protection}

Self-protection is the ability to anticipate, detect, identify and protect against hostile threats from anywhere as they occur and take corrective actions to make them less vulnerable. These activities may be from either internal or external sources and be either accidental or malicious.
Due to internal malfunction or interference, selfprotection quickly works after using intelligent forecast algorithms and problems accurately locating. It will take preventive measures to maximize protection of the entire system.

\subsubsection{Self-healing}

Self-healing is the ability to discover, diagnose and react to faults and disruptions. Self-healing components can detect system malfunctions and initiate policy-based corrective action without disrupting the normal operation. Several possible recovery strategies include the resetting, restarting the component or substituting with another functionally equivalent service that provides the same functional properties as the unavailable service. A key attribute of a self-healing is self-diagnosis that is the ability to diagnose problems from observed symptoms to trigger an automatic reaction. A fault can be the result of a problem in the underlying component, grouping of components or other components in the grid hierarchy. When protection failure or failure cases, self-healing reacts relying on intelligent fault diagnosis and knowledge base. It will also call for self-configuration and self-optimization when necessary to restore APS as soon as possible. Self-healing is the most outstanding specialty that reflects APS autonomic concept.

\section{Autonomic Architecture Design and Analysis}

Based on autonomic concept, APS control and operation architecture is designed with a simple APS situation illustrated in detail.

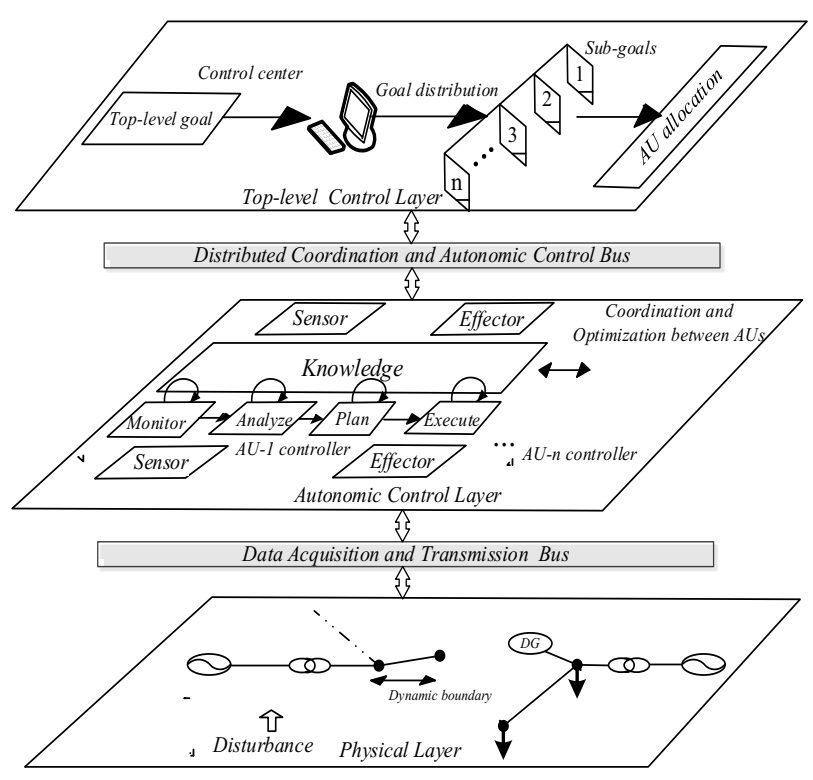

Figure 4. Architecture of APS

As shown in Figure 4, APS operation control architecture is composed by three hierarchies including the top-level control center, autonomic control layer and the physical layer as well as a number of advanced communication infrastructures. Based on goal-directed 
mechanism, the real-time top target is intelligently decomposed into sub-goals at top-level. Then sub-goals are given to appropriate autonomic units (AUs) in autonomic control layer. Based on autonomic computing, each AU will automatically accomplish its current targets and coordination may be needed among them.

As introduced in section 2, an autonomic and coordinating process is designed that contains goaldirected mechanism and dynamic control zones division. Above all, AU plays the most important element in APS architecture. It can be seen that this autonomic architecture will furthest reduce the information interaction between all levels. As shown in figure 5, the inner AU control and operation mechanism is illustrated in detail. The MAPE-cycle (monitoring, analysis, planning and execution) and knowledge base consist of autonomic manager that is the main component of AU. Among them, monitoring and analysis components provide self-awareness and the ability to perceive the external environment. Then planning and execution components enable $\mathrm{AU}$ to make intelligent decisions, adjust current targets, schedule programs and implement.

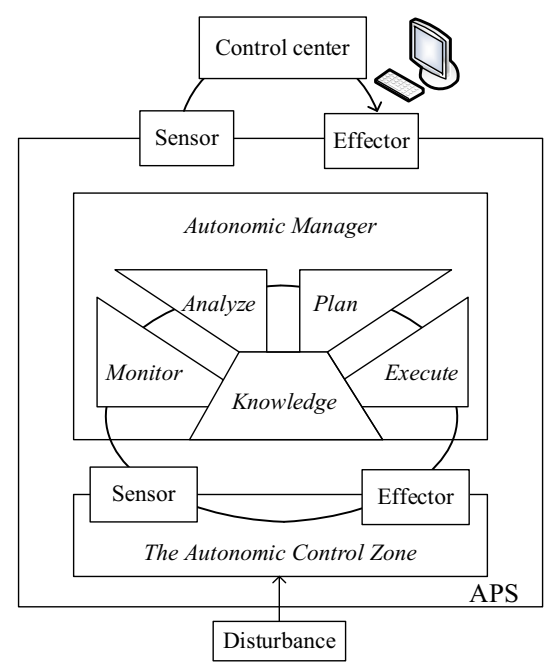

Figure 5. Architecture proposed for autonomic unit

In order to introduce more specific operation mechanism, an APS operation and control flow chart is given in Figure 6, which includes a situation of wind power absorption.

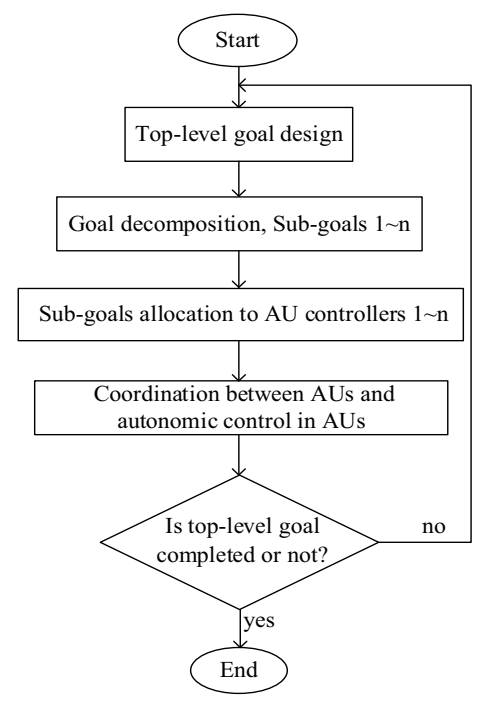

Step 1

Figure 6. APS operation flow chart

The top goal is made by control center. Here, top-level goal is to absorb a certain amount of wind power within a certain time period.

Step 2

Based on APS real-time operation status feedback, toplevel goal is intelligently decomposed into sub-goals $1 \sim \mathrm{N}$ at top-level control center. In this case, sub-goals include reducing power flow from other domains and a certain amount of energy allocation to electric vehicles, etc.

Step 3

Sub-goals $1 \sim \mathrm{N}$ is assigned to corresponding AUs of different functions, such as voltage-autonomic unit, power-autonomic unit.

Step 4

As shown in figure 7, autonomic operation is carried out in each AU MAPE-cycle according to the corresponding objectives, generating corresponding optimal and coordinated programs. Each control objective in $\mathrm{AU}$ is made intelligently based on sub-objectives generated by goal-directed mechanism and monitoring of current operation status in autonomous regions. Optimal autonomous regions at physical layer are produced by AU knowledge base under certain constraints, including the optimal configuration of transmission-distribution network and related electrical equipment. Based on subobjectives and optimal autonomous regions, autonomic and coordination scheme will be executed by AUs, completing current control and operation tasks, such as voltage and reactive power control. 




Figure 7. AUs operation flow chart

\section{Step 5}

As sub-goals completed, an intelligent judgment is made whether overall goal is achieved or not. If not, through the real-time feedback and AU analysis, new commands will be made until previous top-level goal achieved. In this process, related AUs will cooperate coordinately if needed. At the same time, self-configuration, selfoptimization, self-protection and self-healing continually work to make APS overall flexibly and firmly autonomic.

\section{Conclusion}

As a new smart grid, the APS concept is proposed and analyzed in this paper with autonomic architecture designed. Distributed self-management and coordination are two main points in APS. Based on goal-directed mechanism and dynamic control zones division, APS takes an optimally and coordinately autonomic control of power system through AUs autonomy and coordination, with characteristics of self-configuration, selfoptimization, self-protection and self-healing. Reducing the information interaction between layers, APS provides a more advanced and flexible control and operation concept applicable to the power system development. As a prospective and groundbreaking study, research on APS is still in the initial stage with lots of research required, such as distributed constraint optimization, complex network control and advanced information technologies.

\section{ACKNOWLEDGEMENT}

The authors would like to thank the State Grid Corporation of China for providing support to this research work. This prospective project number is XT7114-003.

\section{References}

1. Rahman, S. Green power: what is it and where can we find it. IEEE Power \& Energy Magazine, 1, 1(2003): 30 - 37.

2. Samuelsson O., et al. Active distribution networkDemonstration project ADINE. Innovative Smart Grid Technologies Conference Europe (ISGT Europe), 2010 IEEE PES, IEEE, (2010): 1-8.

3. Ramsay C., D. Pudjianto, and G. Strbac. Taking an active approach. IEEE Power \& Energy Magazine, 5, 4(2007): 68-77.

4. Kephart, J. O., and D. M. Chess. The vision of autonomic computing. Computer, 36, 1(2003): 41-50.

5. Nami, Mohammad Reza, M. Sharifi. A Survey of Autonomic Computing Systems. Intelligent Information Processing III, Springer US, (2007): 101-110.

6. Lopes A. J., R. Lezama, and R. Pineda. Model Based Systems Engineering for Smart Grids as Systems of Systems. Procedia Computer Science, 6(2011): 441-450.

7. Yorino Naoto, et al. An Optimal Autonomous Decentralized Control Method for Voltage Control Devices by Using a Multi-Agent System. IEEE Transactions on Power Systems, 30(2015): 2225-2233.

8. Palizban O, Kauhaniemi K, Guerrero J M. Microgrids in active network management - part II: System operation, power quality and protection. Renewable \& Sustainable Energy Reviews, 36, (C)(2014): 440-451.

9. Piagi, Paolo, R. H. Lasseter. Autonomous control of microgrids. Power Engineering Society General Meeting, 2006, IEEE, (2006): 1-8.

10. Kobayashi H., M. Takasaki. Demonstration Study of Autonomous Demand Area Power System. Transmission and Distribution Conference and Exhibition, 2005/2006 IEEE PES, (2006): 548-555.

11. Rahman, Saifur, M. Pipattanasomporn, and Y. Teklu. Intelligent Distributed Autonomous Power Systems (IDAPS). Power Engineering Society General Meeting, 2007, IEEE, (2007): 1-8.

12. Davidson E. M., et al. AuRA-NMS: An autonomous regional active network management system for EDF energy and SP energy networks. Power and Energy Society General Meeting, 2010 IEEE, (2010): 1 - 6. 